03;01

\title{
Расчет линейного натяжения для простой модели поверхностного нанопузырька
}

\author{
() С.И. Кошоридзе
}

Институт прикладной механики РАН, Москва, Россия

E-mail: koshoridze-semen@yandex.ru

Поступило в Редакцию 22 января 2020 г.

В окончательной редакции 22 января 2020 r.

Принято к публикации 6 февраля 2020 г.

\begin{abstract}
Проведен расчет линейного натяжения для простой модели поверхностного нанопузыря, образованного на гладкой гидрофобной подложке. Показано, что с ростом контактного угла при его определенном критическом значении поверхностное натяжение меняет знак с положительного на отрицательный. Это значит, что оно начинает растягивать нанопузырь, увеличивая его радиус кривизны и тем самым способствуя его стабилизации.
\end{abstract}

Ключевые слова: поверхностный нанопузырь, линейное натяжение, модифицированная формула Юнга, расклинивающее давление.

DOI: 10.21883/PJTF.2020.09.49364.18211

Линейное натяжение $\tau$ на границе твердой, жидкой и газообразной фаз представляет собой избыточную свободную энергию, приходящуюся на единицу длины контакта трех фаз [1]. Для макроскопических капель и пузырьков величина $\tau$ не играет существенной роли, однако при размерах данных объектов порядка сотен нанометров и меньше роль линейного натяжения возрастает.

Основная масса теоретических и экспериментальных работ, касающихся линейного натяжения, посвящена пленкам и сидячим каплям [1-5]. Для поверхностных нанопузырей литературных данных довольно мало, имеются только экспериментальные работы по измерению $\tau$ [6-8] и теоретическая работа [9], посвященная исследованию влияния расклинивающего давления на нанопузыри. С другой стороны, именно для поверхностных нанопузырьков особенно важно знать величину и знак линейного натяжения, так как оно играет большую роль в стабильности этих объектов.

Целью настоящей работы является проведение расчета линейного натяжения поверхностного нанопузырька. Во многом мы будем опираться на работу [9], в которой теоретические методы, развитые для пленок и сидячих капель, впервые были применены к нанопузырькам.

На рис. 1, $a$ приведен схематический баланс сил на границе поверхностного нанопузырька, жидкости (воды) и гидрофобной подложки:

$$
\gamma \cos \varphi+\gamma_{s g}+\frac{\tau}{a}=\gamma_{s w},
$$

где $\gamma, \gamma_{s w}$ и $\gamma_{s g}$ - коэффициенты поверхностного натяжения на границе газ-вода, твердое тело-вода и твердое тело-газ соответственно, $a$ - радиус основания пузырька, $\varphi$ - микроскопический контактный угол. На рис. 1, $a$ показан случай $\tau<0$; при $\tau>0$ вектор $\frac{|\boldsymbol{\tau}|}{a}$ должен поменять направление на противоположное. Термодинамический (макроскопический) контактный угол $\varphi_{0}$, как известно, удовлетворяет классической формуле Юнга:

$$
\cos \varphi_{0}=\frac{\gamma_{s w}-\gamma_{s g}}{\gamma}
$$

Выражения (1), (2) дают модифицированное уравнение Юнга

$$
\cos \varphi=\cos \varphi_{0}-\frac{\tau}{\gamma a}
$$

Таким образом, при $\tau>0$ линейное натяжение сжимает пузырь и выполняется условие $\varphi>\varphi_{0}$. При отрицательных значениях $\tau<0$ пузырь, наоборот, растягивается и контактный угол уменьшается $\left(\varphi<\varphi_{0}\right)$. Уменьшение контактного угла в условиях пиннинга (закрепления границы раздела трех фаз, т.е. постоянства радиуса основания пузырька, $a=$ const) [10] будет способствовать росту радиуса кривизны пузырька $R$ и, как следствие, увеличению его времени жизни из-за уменьшения избыточного лапласовского давления внутри него.

Для простоты расчетов поверхность нанопузырька будем считать сферической (для сидячих капель такое приближение было применено в [11]). Тогда линейное натяжение в условиях пиннинга можно представить в виде интеграла $[5,9]$ :

$$
\tau=\int_{0}^{a}\left[\gamma \sqrt{1+\left(\frac{d h}{d r}\right)^{2}}-\gamma+w(h)-w\left(h_{0}\right)-k p h\right] d r .
$$



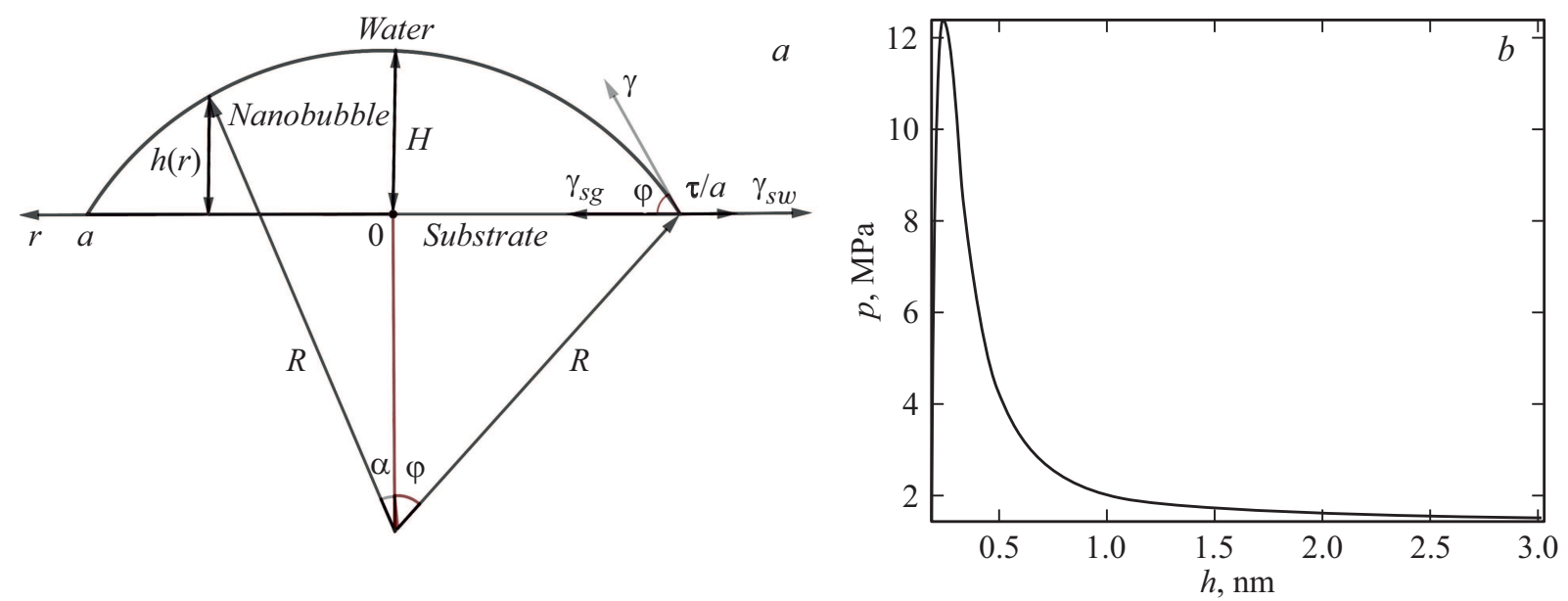

Рис. 1. $a-$ схематическая модель поверхностного нанопузырька на гладкой гидрофобной подложке. $b-$ давление газа $p$ внутри пузырька неоднородно и меняется в зависимости от локальной высоты $h$ нанопузырька. Расчетные параметры: $R=100 \mathrm{~nm}$, $h_{c}=0.2 \mathrm{~nm}, p_{a}=10^{5} \mathrm{~Pa}, \gamma=0.073 \mathrm{~J} / \mathrm{m}^{2}, A_{H}=10^{-20} \mathrm{~J}$.

Здесь

$$
h-h_{c}=\frac{a}{\sin \varphi}(\cos \alpha-\cos \varphi)
$$

- локальная высота нанопузырька, $\quad r=\frac{a}{\sin \varphi} \sin \alpha$, $\frac{d h}{d r}=-\operatorname{tg} \alpha$

$$
w(h)=\frac{A_{H}}{12 \pi h^{2}}\left[\frac{1}{4}\left(\frac{h_{c}}{h}\right)^{6}-1\right]
$$

- энергия ван-дер-ваальсовского взаимодействия (на единицу площади) молекул воды и твердого тела через газовую щель толщины $h$, записанная в модели Леннарда-Джонса,

$$
\Pi=-\frac{d w}{d h}=\frac{A_{H}}{6 \pi h^{3}}\left[\left(\frac{h_{c}}{h}\right)^{6}-1\right]
$$

- так называемое расклинивающее давление, $A_{H}>0-$ постоянная Гамакера, $h_{c}$ - толщина, при которой потенциал $w(h)$ достигает минимума. При $h>h_{c}$ преобладает притяжение между молекулами подложки и воды, и расклинивающее давление П $(h)$ отрицательно. При $h<h_{c}$ преобладает отталкивание, и П $(h)$ положительно. Величину $h_{c}$ часто называют длиной среза; при данной толщине расклинивающее давление равно нулю: $\Pi\left(h_{c}\right)=0$. В (5) сделано предположение [9], что у края нанопузырька остается очень тонкая газовая прослойка толщины $h_{c}$, из-за чего толщина щели $h$ там не равна нулю, и выражения (6) и (7) остаются конечными (на рис. 1 из-за соотношения $h_{c} / H \ll 1$ эта прослойка не показана).

Давление внутри нанопузырька $p(h)$ неоднородно (зависит от координаты $r$ ) и удовлетворяет дифференциальному уравнению [9]:

$$
p \ln \left(\frac{p}{p_{0}}\right)=-\Pi(h)
$$

Здесь $p_{0}=p_{a}+(2 \gamma / R) \quad$ - давление внутри пузырька при отсутствии расклинивающего давления $($ т.е. при $h \rightarrow \infty), p_{a}-$ внешнее давление, равное для определенности атмосферному. В результате численного решения (8) получается график, представленный для наглядности на рис. $1, b$. Максимум давления соответствует $h \approx 1.2 h_{c}$. Давление газа зависит от расстояния до оси симметрии $r$ : минимально при $r=0$ (там, где высота пузырька максимальна, $h=H$ ) и растет при увеличении $r$ (уменьшении $h$ ) вследствие роста влияния ван-дер-ваальсовского взаимодействия.

В подынтегральном выражении (4) фигурирует константа

$$
k=-\frac{\mu}{k_{\mathrm{B}} T},
$$

где $\mu-$ свободная энергия Гиббса в расчете на одну молекулу газа (одинаковая для всех молекул внутри пузырька), $k_{\mathrm{B}}$ - постоянная Больцмана, $T-$ абсолютная температура. $k$ играет роль параметра теории.

Для определения $k$ используется граничное условие [9]:

$$
\frac{2 \gamma}{R}=-\left.\frac{d U}{d h}\right|_{h=H}
$$

где

$$
U(h)=w(h)-w\left(h_{0}\right)-k p h
$$

- так называемый эффективный потенциал,

$$
H=R(1-\cos \varphi)
$$

- высота нанопузырька. После подстановки (9) и (11) в (10) получаем

$$
k=\frac{(2 \gamma / R)+w^{\prime}(H)}{p(H)+H p^{\prime}(H)} .
$$

Если учесть, что $w^{\prime}(H)=-\Pi(H)>0 \quad$ (см. (7)) и $p^{\prime}(H)<0$ (рис. $\left.1, a\right)$, можно написать

$$
k \geq k_{\min } \text {. }
$$


Здесь

$$
k_{\min }=\frac{2 \gamma / R}{p(H)} \approx \frac{2 \gamma / R}{p_{a}+(2 \gamma / R)}=\frac{2 \gamma \sin \varphi}{a p_{a}+2 \gamma \sin \varphi} .
$$

Второе неравенство в (15) означает, что при $h=H$ расклинивающим давлением можно пренебречь.

На рис. 2 приведен результат расчета линейного натяжения $\tau$ от контактного угла $\varphi$ в условиях пиннинга $(a=$ const). Показано, что при критическом угле $\varphi_{c}=18^{\circ}$ линейное натяжение меняет знак.

На рис. 3 приведена зависимость величины $\left(\varphi_{0}-\varphi\right)$ от $\varphi$, рассчитанная с помощью (3). Как видно, при $\varphi>\varphi_{c}=18^{\circ}$ линейное натяжение существенно уменьшает микроскопический угол $\varphi$ по сравнению с макроскопическим $\varphi_{0}$. Таким образом, при $\varphi<\varphi_{c}$ линейное натяжение увеличивает контактный угол $\left(\varphi>\varphi_{0}\right)$, а при $\varphi>\varphi_{c}-$ уменьшает его $\left(\varphi<\varphi_{0}\right)$. В условиях пиннинга в первом случае линейное натяжение стремится уменьшить радиус кривизны пузырька $R$, а во втором случае стремится его увеличить и способствует росту времени жизни нанопузырька.

Отметим, что похожее явление - смена знака линейного натяжения $\tau$ с ростом макроскопического контактного угла $\varphi_{0}$ с положительного на отрицательный - было обнаружено недавно с помощью численного моделирования и подтверждено экспериментально для сидячих нанокапель в [12].

Таким образом, при использовании упрощенной модели поверхностного нанопузырька впервые удалось рассчитать линейное натяжение в области границы трех фаз. Показано, что знак линейного натяжения $\tau$ с ростом микроскопического контактного угла $\varphi$ меняется с положительного на отрицательный. Отрицательный знак

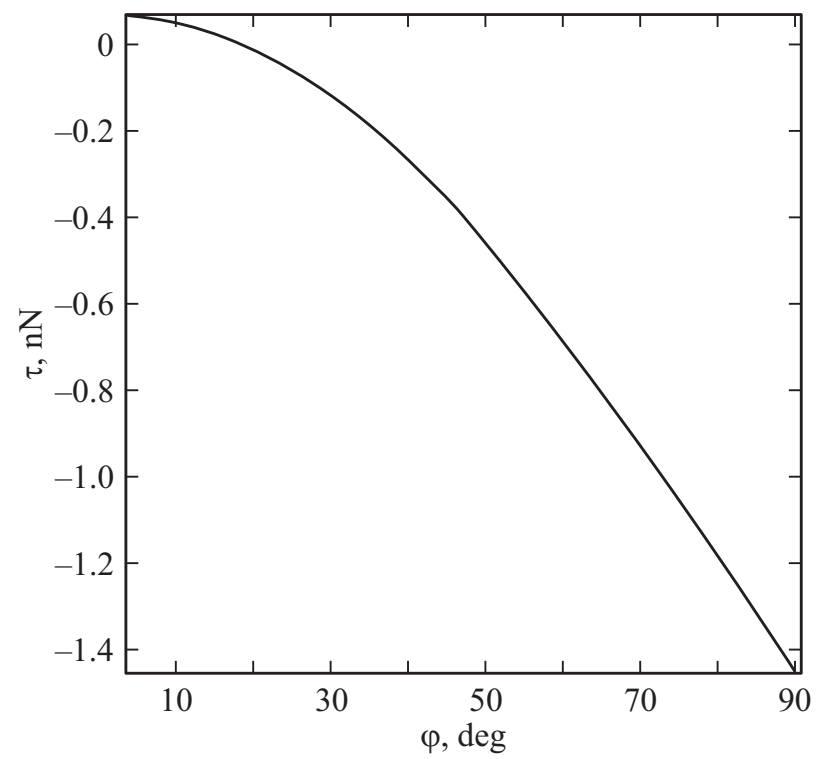

Рис. 2. Зависимость линейного натяжения $\tau$ от контактного угла $\varphi$ в условиях пиннинга. Расчетные параметры: $a=20 \mathrm{~nm}$, $k=1$. Остальные параметры те же, что на рис. $1, b$ (кроме радиуса нанопузырька $R=a / \sin \varphi$, который на данном рисунке менялся).

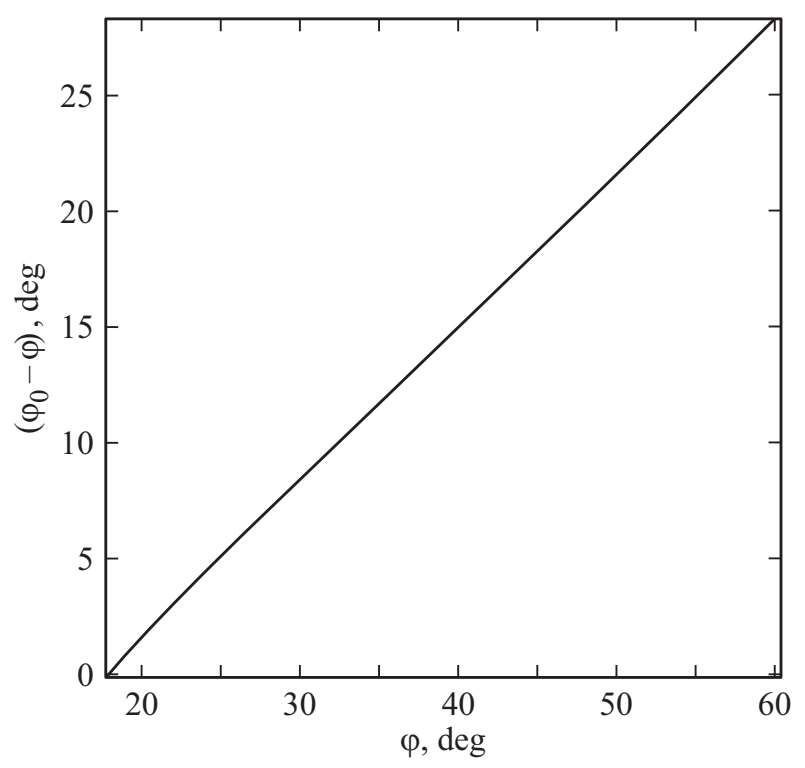

Рис. 3. Зависимость разности между макро- и микроскопическими значениями контактного угла $\left(\varphi_{0}-\varphi\right)$ от микроскопического контактного угла $\varphi$. Расчетные параметры идентичны использованным для рис. 2 .

линейного натяжения при $\varphi>\varphi_{c}$ указывает на существенную роль величины $\tau$ в обеспечении стабильности поверхностного нанопузырька.

\section{Конфликт интересов}

Автор заявляет, что у него нет конфликта интересов.

\section{Список литературы}

[1] Widom B. // J. Phys. Chem. 1995. V. 99. P. 2803-2806. https://doi.org/10.1021/j100009a041

[2] Drelich J.W. // Adv. Colloid Interface Sci. 2019. V. 267. P. 1-14. https://doi.org/10.1016/j.cis.2019.02.002

[3] Kanduc M., Eixeres L., Liese S., Netz R.R. // Phys. Rev. E. 2018. V. 98. P. 032804. DOI: 10.1103/PhysRevE.98.032804

[4] Drelich J. // Colloids Surf. A. 1996. V. 116. P. 43-54. https://doi.org/10.1016/0927-7757(96)03651-5

[5] Solomentsev Y., White L.R. // J. Colloid Interface Sci. 1999. V. 218. P. 122-136. https://doi.org/10.1006/jcis.1999.6389

[6] Kameda N., Nakabayashi S. // Chem. Phys. Lett. 2008. V. 461. P. 122-126. DOI: 10.1016/j.cplett.2008.07.012

[7] Kameda N., Sogoshi S., Nakabayashi S. // Surf. Sci. 2008. V. 602. P. 1579-1584. DOI: 10.1016/j.susc.2008.02.023

[8] Yang J., Duan J., Fornasiero D., Ralston J. // J. Phys. Chem. B. 2003. V. 107. P. 6139-6147. DOI: 10.1039/B709624K

[9] Svetovoy V.B., Devic I., Snoeijer J.H., Lohse D. // Langmuir. 2016. V. 32. P. 11188-11196. DOI: 10.1021/acs.langmuir.6b01812

[10] Lohse D., Zhang X. // Phys. Rev. E. 2015. V. 91. P. 031003. DOI: 10.1103/PhysRevE.91.031003

[11] Marmur A. // J. Colloid Interface Sci. 1997. V. 186. P. 462-466. https://doi.org/10.1006/jcis.1996.4666

[12] Zhao B., Luo S., Bonaccurso E., Auernhammer G.K., Deng X., Li Z., Chen L. // Phys. Rev. Lett. 2019. V. 123. P. 094501. DOI: 10.1103/PhysRevLett.123.094501 